\title{
La « querelle des vernis », une controverse sur le nettoyage des peintures au milieu du $\mathrm{XX}^{\mathrm{e}}$ siècle
} The "cleaning controversy", a mid-twentieth-century controversy over the cleaning of paintings

\section{Fanny Girard}

\section{(2) OpenEdition}

\section{Journals}

Édition électronique

URL : http://journals.openedition.org/cel/4458

DOI : $10.4000 /$ cel. 4458

ISSN : 2262-208X

Éditeur

École du Louvre

Référence électronique

Fanny Girard, "La « querelle des vernis », une controverse sur le nettoyage des peintures au milieu du XXe siècle », Les Cahiers de l'École du Louvre [En ligne], 14 | 2019, mis en ligne le 02 décembre 2019 consulté le 05 décembre 2019. URL : http://journals.openedition.org/cel/4458 ; DOI : 10.4000/cel. 4458

Ce document a été généré automatiquement le 5 décembre 2019

\section{(i) $\$$

Les Cahiers de l'École du Louvre sont mis à disposition selon les termes de la licence Creative Commons Attribution - Pas d'Utilisation Commerciale - Pas de Modification 4.0 International. 


\title{
La « querelle des vernis », une controverse sur le nettoyage des peintures au milieu $\mathrm{du} \mathrm{XX}^{\mathrm{e}}$ siècle
}

\author{
The "cleaning controversy", a mid-twentieth-century controversy over the \\ cleaning of paintings
}

Fanny Girard

1 La "querelle des vernis", polémique se développant entre 1946 et 1963 autour du traitement des vernis des peintures, témoigne d'un moment de réflexion intense sur la restauration et les degrés d'intervention sur les œuvres. Moment marquant de l'histoire de la restauration, son analyse permet de souligner son importance dans la mise en place des principes actuels de conservation et de restauration des biens culturels. L'analyse de cette controverse s'est fondée sur près d'une centaine de textes publiés durant la querelle, principalement des lettres de critiques et des articles de différents journaux et revues, et sur l'étude des textes historiographiques et de l'image qu'ils donnent de cette polémique. L'examen de ces sources premières a permis de nuancer et d'apporter des précisions, mais également de distinguer les apports de cette controverse. En effet, la dimension conflictuelle ne doit pas occulter la collaboration internationale et interdisciplinaire qu'a développée la «querelle des vernis » et les progrès qu'elle a permis, notamment en termes de prudence des interventions, de dialogue, de documentation et de formation des restaurateurs. Couvrant la période de 1946 à 1963, elle précède directement la théorisation de la conservation-restauration moderne et le lien entre les deux ne peut manquer d'être établi au regard des nombreux points débattus élevés par la suite au rang de principes clés et des acteurs éminents intervenant dans cette controverse. Elle voit s'opposer tant les grandes institutions mondiales, la National Gallery de Londres et le Louvre en tête, que différents conservateurs, historiens de l'art, restaurateurs et scientifiques qui échangent leur point de vue dans des institutions internationales ou au moyen d'articles interposés. Si cette controverse est liée à certaines personnalités connues telles que Cesare Brandi, René Huyghe ou Ernst Gombrich, son analyse a contribué 
également à souligner le rôle essentiel de nombreux autres acteurs. De leurs débats émergent des notions fondatrices des pratiques actuelles, donnant à cette polémique une place majeure dans l'histoire de la restauration mais aussi de la conservation des biens culturels.

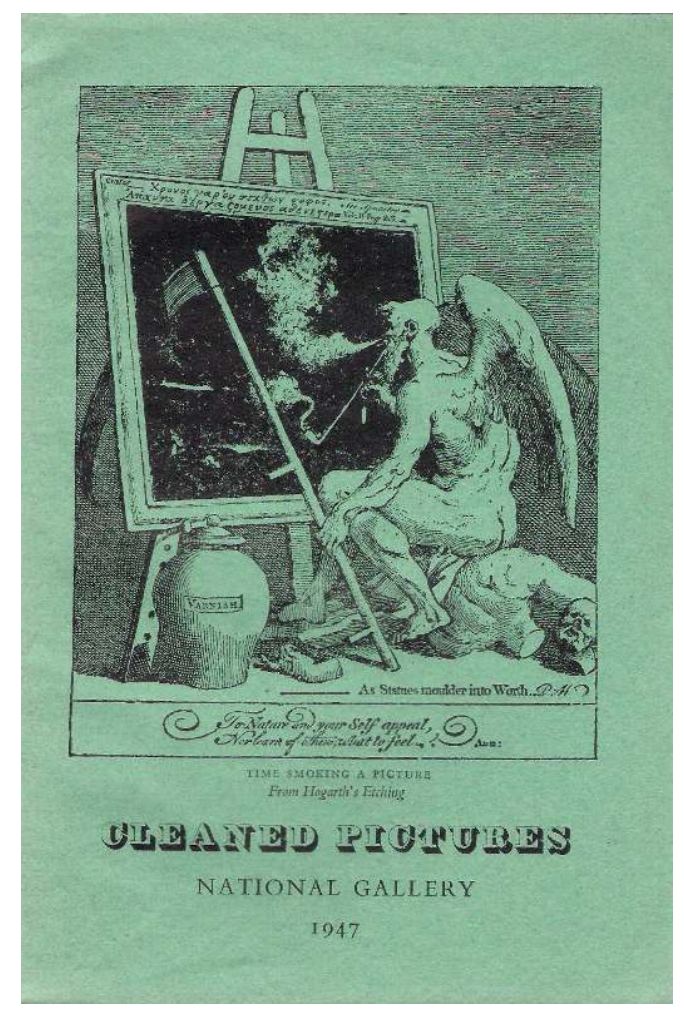

Cat. d'exp. An exhibition of cleaned pictures, 1936-1947, sous la direction de Sir Philip Hendy, Londres, The National Gallery, 1947, Londres, The National Gallery, 1947

\section{Une polémique en plusieurs phases}

Développée sur près d'une vingtaine d'années, la « querelle des vernis » connaît trois phases, intégrant chacune des acteurs, des débats et des dimensions différentes. Elle débute en 1946 à la National Gallery de Londres et s'inscrit dans une tradition de polémiques portant sur le nettoyage des peintures ${ }^{1}$, survenant un siècle après une première controverse de grande ampleur survenue dans cette institution de 1846 à 1853 et une autre simultanée au musée du Louvre²

\section{La controverse autour des tableaux nettoyés de la National Gallery de Londres}

Durant la Seconde Guerre mondiale, profitant de l'envoi des œuvres hors de Londres loin des bombardements et du regard du public, les autorités de la National Gallery décident de faire nettoyer une partie de sa collection ${ }^{3}$. Une fois la guerre finie, les tableaux traités sont exposés à la réouverture de la National Gallery au milieu d'œuvres non nettoyées. Leurs teintes plus claires et froides, dues à la suppression du vernis jauni, contrastent fortement avec celles des autres œuvres, au point de soulever une vague de critiques: plus d'une trentaine de lettres portant sur ces nettoyages sont 
publiées dans le Times entre le 30 octobre 1946 et le 15 mai $1947^{4}$. Rédigées principalement par des artistes, des historiens de l'art et des personnalités appartenant au domaine muséal, ces lettres sont majoritairement négatives et dénoncent le nettoyage trop poussé allant jusqu'au dévernissage total des peintures. Ces critiques reposent essentiellement sur une impression personnelle due aux conditions de présentation. En effet, les visiteurs de la National Gallery, habitués à voir les œuvres recouvertes de leur vernis sombre et jauni, ont été surpris par leur nouvel aspect et leurs couleurs vives révélées par le nettoyage. À cette surprise s'ajoutent des préjugés particuliers à la culture occidentale qui dénigrent et marginalisent les couleurs vives, phénomène que David Batchelor appelle la "chromophobie ${ }^{5}$, mais également une prise de conscience accrue, au lendemain de la Seconde Guerre mondiale, de la nécessité de protéger le patrimoine national. Quelques critiques positives sont également formulées, tandis que certains auteurs développent une réflexion plus poussée et formulent des demandes précises pour contrôler les nettoyages tels que la création d'une école de restaurateurs, la mise en place d'une commission d'enquêtes sur les dommages causés par les nettoyages ou le développement d'une plus grande transparence envers le public par la mise à disposition de la documentation sur les nettoyages ou l'exposition de tous les tableaux nettoyés récemment.

En réaction aux critiques, les autorités de la National Gallery mettent en place un comité spécial d'investigation pour enquêter sur la réalité des dommages décriés. Présidé par John Reginald Homer Weaver, ce comité est composé de deux experts étrangers extérieurs à l'institution, George Leslie Stout et Paul Coremans ${ }^{6}$. Leur enquête se concentre sur les dix tableaux dont le nettoyage avait fait l'objet de critiques dans la presse, et débouche sur la publication du "rapport Weaver $»^{7}$. Celui-ci conclut qu'aucune œuvre a été matériellement abîmée par son nettoyage et présente également des recommandations générales en matière de conservation et de nettoyage.

La diffusion de ce rapport restant cependant limitée, les autorités de la National Gallery décident de se justifier face au grand public en réalisant une exposition, intitulée $A n$ exhibition of cleaned pictures. Débutant en octobre 1947, elle présente tous les tableaux de la National Gallery qui ont été nettoyés durant les dix dernières années, accompagnés de nombreuses photographies avant, pendant et après nettoyage, sous différents rayonnements. L'objectif de cette manifestation est de prouver que le nettoyage n'a fait subir aucun dommage aux œuvres et, par conséquent, de louer la pratique de dévernissage de la National Gallery. Elle s'accompagne d'un catalogue ${ }^{8}$ dont l'avantpropos, rédigé par le directeur de la National Gallery Sir Philip Hendy, répond à toutes les critiques formulées antérieurement dans le Times. Attirant des spécialistes du monde entier, elle soumet à leur réflexion le problème du nettoyage des peintures et de sa réception par le public, faisant ainsi naître questionnements et débats.

\section{Le développement du débat à une échelle internationale}

5 Consécutivement à la polémique et à l'exposition de la National Gallery, le problème du nettoyage des peintures est abordé lors de la première conférence biennale du Conseil International des Musées (ICOM), dans un groupe de travail dédié aux questions de restauration et présidé par René Huyghe, conservateur en chef du département des peintures du Musée du Louvre. Est alors décidé de créer une commission spéciale 
consacrée à la question du nettoyage des peintures ${ }^{9}$, pour fournir un cadre de réflexion sur ce problème et chercher des solutions pour éviter les critiques et les polémiques.

Adoptant le nom de Commission de l'ICOM pour le traitement des peintures, ce comité international est dans un premier temps restreint aux directeurs et aux conservateurs de musées. Les premières conférences se concentrent sur le problème du nettoyage, en lien avec la récente polémique. En effet, la conférence initiale a lieu à la National Gallery de Londres, du 13 au 15 décembre 1948, et traite exclusivement des méthodes de nettoyage ${ }^{10}$, en s'appuyant notamment sur une étude des tableaux exposés dans An Exhibition of cleaned pictures. La deuxième conférence, qui a lieu un an plus tard à l'Istituto Centrale del Restauro de Rome, étudie de nouveau la question et cherche une solution concrète pour limiter la fréquence et la violence des polémiques. Elle s'appuie pour cela sur les réponses à des questionnaires, diffusés durant l'année à l'ensemble des grandes institutions mondiales, portant sur leurs pratiques et principes de restauration. Cette commission crée ainsi un cadre propice au dialogue constructif d'experts sur les questions de restauration et de nettoyage des œuvres.

6 Si les conférences suivantes semblent s'écarter du sujet du nettoyage, les débats de cette commission sont diffusés à une échelle internationale dans la presse, permettant d'élargir ainsi le débat hors du cercle restreint des membres de la commission. Deux numéros de la revue Museum de l'Unesco (vol. III, n ${ }^{\text {os } 2}$ et 3) sont consacrés en 1950 à la question du dévernissage, de même qu'un numéro spécial d'Alumni (vol. XIX, $\mathrm{n}^{\text {os }}{ }^{3}-4$ ), revue universitaire belge. Les articles de ces numéros présentent les points de vue sur le nettoyage et les pratiques de dévernissage et d'allègement de vernis de différents experts, pour la majorité membre de la commission de l'ICOM pour le traitement des peintures. C'est notamment dans un des numéros de Museum que René Huygue théorise la pratique française de l'allègement de vernis ${ }^{11}$. Ils opposent ainsi essentiellement deux pratiques concurrentes de nettoyage : le dévernissage total retirant toute trace de l'ancien vernis et l'allègement de vernis qui laisse subsister une fine couche à la surface. Touchant principalement des professionnels, ces revues diffusent le débat, à une échelle néanmoins restreinte.

\section{L'élargissement du débat}

7 D'autres publications permettent de porter à la connaissance d'un plus large public le débat sur le nettoyage des peintures. En effet, plus d'une vingtaine d'articles paraissent entre 1949 et 1963, principalement dans The Burlington Magazine et The British Journal of Aesthetics $^{12}$. Ils explorent des problématiques plus précises et complexes que la promotion des bienfaits ou le soulignement des défauts du dévernissage total ou de l'allègement de vernis, se distinguant ainsi des articles publiés dans Museum et Alumni. En effet, la grande majorité des auteurs s'interroge sur des notions plus techniques en lien avec le traitement du vernis, comme celles de la patine, des glacis et des vernis teintés. Des oppositions se développent autour de ces concepts, engendrant un véritable débat transcrit dans une longue suite d'articles se répondant et essayant de se réfuter les uns les autres.

8 Si parmi les auteurs se retrouvent quelques noms de membres de la commission de l'ICOM pour le traitement des peintures tels que Cesare Brandi et Neil MacLaren, le grand intérêt de cette nouvelle phase de la «querelle des vernis" est de faire intervenir des personnalités nouvelles et très diverses. La majorité des auteurs sont en 
effet des historiens de l'art, des restaurateurs et des scientifiques qui, ne pouvant intervenir dans la commission de l'ICOM réservée aux conservateurs, s'expriment dans la presse. L'intervention de spécialistes de différents domaines entraîne des oppositions fortes, notamment entre historiens de l'art et scientifiques, illustrant un problème de compréhension mutuelle. Transparaît également un conflit entre les institutions employant ces experts : à la National Gallery de Londres, représentée entre autres par le restaurateur Helmut Ruhemann et la scientifique Joyce Plester, semble s'opposer l'Istituto Centrale del restauro de Cesare Brandi, le Warburg Institute incarné par Otto Kurz et Ernst Gombrich et le Courtauld Institute.

Le débat s'ouvre ainsi aux autres domaines intrinsèques à la conservation et la restauration des biens culturels et dépasse les questionnements initiaux sur le dévernissage des tableaux pour aborder d'autres aspects. En étudiant des notions précises liées au nettoyage au prisme des spécialités respectives de leurs auteurs, ces articles permettent de développer les connaissances sur des domaines en marge de la controverse opposant dévernissage et allègement de vernis.

Dépassant le stade d'une simple polémique suscitée par le dévernissage d'un ou plusieurs tableaux, la " querelle des vernis » se développe en débats sur les principes et pratiques du nettoyage des peintures et crée un cadre où s'opposent des acteurs divers. L'historiographie de cette controverse en a fait un épisode connu de l'histoire de la restauration et l'a essentiellement présentée comme le cadre de fortes oppositions en soulignant trois conflits majeurs, considération qui est cependant à nuancer.

\section{L'image caricaturale d'une polémique à trois visages}

9 Fréquemment mentionnée dans les écrits relatifs à l'histoire de la restauration, l'appréhension de la «querelle des vernis» est complexifiée par la présentation caricaturale qui en est souvent donnée, celle d'une opposition radicale entre deux "écoles de nettoyage ${ }^{13}$ »: d'une part les «nettoyeurs totalitaires» partisans du dévernissage total des peintures, et de l'autre les «nettoyeurs nuancés» qui prônent l'allègement de vernis ${ }^{14}$. Cette confrontation transparaît particulièrement dans les écrits de spécialistes français, comme Gilberte Emile-Mâle et Ségolène Bergeon Langle ${ }^{15}$, qui intègrent généralement la querelle dans une argumentation en faveur de l'allègement de vernis, René Huyghe ayant théorisé cette pratique dans le cadre de cette controverse. À ce premier conflit s'ajoutent deux autres oppositions : une dispute entre pays anglo-saxons et pays latins, les premiers se rattachant aux «nettoyeurs totalitaires " et les seconds aux "nuancés ", ainsi qu'une confrontation entre une pratique de nettoyage reposant sur des critères scientifiques et une autre fondée sur des critères esthétiques. Ces trois dimensions sont étroitement liées. Ainsi, les "nettoyeurs totalitaires» sont assimilés aux Anglo-saxons et au nettoyage dit scientifique, tandis que les "nuancés » sont rapprochés des Latins et du nettoyage dit esthétique. Les débats soulevés par la " querelle des vernis » dépassent cependant cette présentation. Si chacun de ces trois conflits transparaît en effet au premier abord, l'étude détaillée de la « querelle des vernis » a permis de les nuancer. 


\section{Une vision à nuancer : le conflit entre les « nettoyeurs totalitaires » et les « nettoyeurs nuancés »}

10 Cette opposition constitue le conflit principal et le cœur de la "querelle des vernis ». Néanmoins, l'étude de l'ensemble des arguments échangés par les acteurs de la controverse, sans se focaliser sur la justification d'une seule des deux pratiques, a permis d'en donner une image plus nuancée. Souvent opposés radicalement dans l'historiographie, il s'avère que les adversaires s'accordent en fait sur certains points et se rapprochent dans la pratique.

11 Le dévernissage total et l'allègement de vernis reposent en effet sur des objectifs proches voire similaires, mais témoignent d'une différence de conception débouchant sur ces deux procédés antagonistes. Ainsi, les partisans du dévernissage total comme ceux de l'allègement de vernis prônent dans leurs articles la prudence comme critère indispensable pour ne pas abîmer l'œuvre, mais répondent différemment à ce critère. Les «nettoyeurs nuancés » laissent une couche de vernis afin de ne pas mettre à nu la couche picturale et risquer de retirer des glacis originaux ${ }^{16}$, tandis que les «nettoyeurs totalitaires " louent le retrait de la totalité du vernis pour limiter la fréquence des nettoyages et donc le risque encouru par l'œuvre au cours de cette opération ${ }^{17}$. La finalité est donc identique mais les moyens différents. Les deux clans préconisent par ailleurs de ne pas intervenir en cas de doute et d'attendre le développement de techniques plus sûres. Ils justifient également leur pratique avec les mêmes critères de respect de l'intention de l'artiste. Ainsi, les «nettoyeurs totalitaires » retirent tout le vernis pour retrouver les couleurs originales telles qu'appliquées par le peintre ${ }^{18}$, tandis que les "nuancés » considèrent que l'état original est perdu en raison de l'évolution avec le temps des constituants de l'œuvre ${ }^{19}$ et qu'un voile de vernis laissé à la surface, en atténuant les désaccords de teintes dues au temps, permet de rendre l'harmonie de l'œuvre telle que voulue par l'artiste ${ }^{20}$. Ils s'appuient aussi tous deux sur le respect des éléments originaux et de la technique de l'artiste mais une différence de conception de la nature du vernis oppose leurs pratiques. Les " nettoyeurs totalitaires » ne considèrent pas le vernis comme un constituant de l'œuvre mais comme un apport indésirable qu'il faut donc retirer ${ }^{21}$, à l'inverse des «nuancés" pour qui son retrait reviendrait à éliminer un élément original appliqué par l'artiste.

Les deux approches suggèrent donc des pratiques antagonistes, mais les deux clans se rapprochent en réalité dans la pratique. En effet, ils prônent de façon commune l'adaptation au cas particulier et par conséquent n'hésitent pas dans les faits à appliquer la pratique opposée à leurs convictions, rendant ainsi caduque l'affirmation d'un antagonisme radical entre les deux conceptions de nettoyage.

\section{Une vision à préciser : le conflit entre les pays anglo-saxons et les pays latins}

12 L'opposition entre les Anglo-saxons et les Latins, identifiée dans de nombreuses publications comme un conflit entre les Anglais d'une part et les Italiens et les Français d'autre part, ne rend pas compte de la réalité des débats de la "querelle des vernis ", qui met en scène de nombreux autres pays. En effet, l'historiographie se focalise souvent sur les grands noms de l'histoire de l'art et de l'histoire de la restauration intervenant dans cette controverse, comme Cesare Brandi, René Huyghe et Ernst 
Gombrich, et occulte les autres acteurs. Ce phénomène est d'autant plus dommageable que certains acteurs et certains pays sont particulièrement actifs dans le débat, notamment la Belgique avec la forte implication de Paul Coremans, Paul Fierens et René Sneyders.

13 Simplificatrice, l'identification d'un conflit entre pays anglo-saxons et pays latins ne peut refléter une réalité forcément plus complexe, et résulte d'une focalisation sur un nombre restreint d'institutions telles que le Louvre, la National Gallery de Londres, l'Istituto Centrale del Restauro de Rome ou encore l'Institut Royal du Patrimoine Artistique de Bruxelles. Certaines sources font même de la "querelle des vernis » un duel entre le Louvre et la National Gallery ${ }^{22}$, vision trop caricaturale mais qui témoigne cependant d'une réelle concurrence entre les deux institutions. Ce concours de réputation est reflété par un certain nombre de remarques critiques inscrites dans les procès-verbaux des commissions de restauration ayant eu lieu au musée du Louvre pendant la période de la $"$ querelle des vernis $»^{23}$. Cette controverse ne peut cependant se restreindre à ce duel, ce serait oublier les autres acteurs et nier la pluralité de pratiques de chaque pays. Au sein d'un même territoire se développent en effet des pratiques différentes au gré des institutions et de leur personnel. Ainsi, si la National Gallery prône bien le dévernissage total, cette pratique va à l'encontre de celle de la Tate Gallery qui se rapproche davantage de l'allègement de vernis ${ }^{24}$, mais aussi des opinions du Warburg Institute et du Courtauld Institute de Londres formulées dans les articles mentionnés précédemment.

14 La «querelle des vernis » semble en définitive être davantage un conflit entre personnalités qu'un conflit entre pays latins et anglo-saxons ou entre institutions étrangères, laissant supposer des luttes d'ego. Si l'assimilation de la «querelle des vernis » à un " débat Ruhemann-Gombrich ${ }^{25}$ " par Steven W. Dykstra semble exagérée au regard de la faible occurrence de ces acteurs, il convient cependant de noter que la polémique est marquée par des tensions particulièrement fortes entre le directeur de la National Gallery, Sir Philip Hendy, et les conservateurs du Louvre, René Huyghe puis Germain Bazin. La vision englobante d'un conflit entre pays anglo-saxons et pays latins est ainsi à préciser.

\section{Une vision à relativiser : le conflit entre les défenseurs du « nettoyage scientifique objectif » et ceux du « nettoyage esthétique subjectif »}

15 Cette dernière dimension de la polémique illustre un rapport différent à la science et de ce fait à son implication dans la conservation et la restauration des œuvres. Deux tendances se dégagent, que l'historiographie a relié aux clans distingués précédemment ${ }^{26}$. La première consiste à accorder à la science une confiance totale : les "nettoyeurs totalitaires anglo-saxons » se reposent pour effectuer leurs nettoyages sur la croyance qu'un contrôle scientifique limite le danger encouru par l'œuvre, mais ils restent cependant conscients que le risque d'erreur persiste. À l'inverse, la seconde tendance s'apparente à un fort scepticisme : les «nuancés latins » se méfient de l'aide qu'apportent les nouveautés scientifiques, sans cependant exclure leur usage, et préfèrent fonder leurs opérations de nettoyage sur des critères esthétiques.

16 Par conséquent, les divergences de pratiques de nettoyage résultent des « différences dans l'appréciation de certains arguments d'ordre esthétique et de faits matériels 
précis $^{27}$ ». Arguments esthétiques et scientifiques ne sont pas à opposer radicalement : chaque clan les prend en compte lors de leur choix de pratiques de nettoyage. Celui-ci est motivé par une sensibilité plus importante pour l'un ou l'autre type d'argument mais est également fondé sur une étude de leur valeur effective pour chaque intervention particulière. Ainsi, chaque nettoyage, «scientifique " comme "esthétique ", s'appuie sur l'évaluation de preuves scientifiques et esthétiques et de justifications reposant sur des écrits anciens et des preuves matérielles visuelles ${ }^{28}$. Leur différence repose sur une question de préférence, que l'on pourrait assimiler à une prédilection pour la matière de l'œuvre ou pour son image. Ainsi, ce conflit se matérialise dans le comportement adopté face à la patine: selon Cesare Brandi, la patine «amortit la présence de la matière dans l'œuvre d'art ${ }^{29}$ " et permet ainsi de privilégier l'image. Le choix des "nuancés» de conserver la patine en laissant une couche de vernis à la surface des œuvres vise donc à donner la prééminence à l'image sur la matière, tandis que le dévernissage total, en mettant à nu la couche picturale, tend à souligner la matérialité de l'œuvre.

17 À cette confrontation entre nettoyages dits «scientifique » et "esthétique " s'est ajoutée la question de l'objectivité des pratiques. L'allègement de vernis des «nuancés latins » repose en partie sur une sensibilité du restaurateur qui décide quand l'arrêter selon l'aspect visuel souhaité. Pour autant, le dévernissage défendu par les « nettoyeurs totalitaires anglo-saxons " peut également être considéré comme subjectif. En effet, il résulte d'un choix de dévernir totalement qui n'est pas indépendant du goût contemporain pour des peintures plus claires. Ainsi, d'après Paul Philippot, « on a pu, non sans raison, voir dans les nettoyages des années 1940 une projection de chromatismes expressionnistes ${ }^{30}$ ». L'objectivité du nettoyage apparait de ce fait illusoire puisque celui-ci est déterminé par une recherche de l'état original, lequel est imaginé par le restaurateur et par conséquent influencé par les goûts et pratiques contemporains. L'opposition entre "nettoyage scientifique objectif» et "nettoyage esthétique subjectif» évoquée dans certains textes historiographiques est ainsi également à relativiser.

Les trois dimensions du conflit incluses dans les débats de la «querelle des vernis » et relevées par l'historiographie sont donc à nuancer, d'autant plus que cette controverse n'est pas seulement le cadre d'affrontements mais également celui de recherche de solutions au problème du nettoyage des peintures.

\section{Une polémique essentielle pour la mise en place de certains principes clés de la conservation-restauration actuelle}

18 La «querelle des vernis » a engendré des réflexions sur différents aspects aujourd'hui considérés comme des principes clés de la conservation-restauration, en premier lieu l'ouverture à la collaboration. Des critiques sur le nettoyage des peintures de la National Gallery de Londres découle en effet une prise de conscience de la nécessité de réfléchir au problème du nettoyage des tableaux, non plus au sein de chaque institution indépendamment mais en développant un dialogue entre les experts de différentes nationalités et de différents domaines. Afin de déterminer de bonnes pratiques de nettoyage et de limiter la fréquence des polémiques, une collaboration internationale et interdisciplinaire apparaît et se développe lors de la « querelle des vernis». 


\section{L'essor de la coopération internationale}

19 discussion et une réflexion internationales sur les questions de nettoyage des peintures et plus généralement de restauration. Elle se développe en effet en partie dans des structures internationales comme l'ICOM et sa commission pour le traitement des peintures, et confronte ainsi dans ses débats des experts de diverses nationalités. Conscients de la nécessité de développer une réflexion commune pour trouver des solutions au problème récurrent des polémiques sur le nettoyage, ces derniers œuvrent à restaurer la collaboration internationale au lendemain de la Seconde Guerre mondiale.

Se penchant d'abord sur les conditions pratiques nécessaires à la mise en place d'un dialogue international efficace, ils s'accordent sur une nécessité: "pour éviter l'équivoque, si fréquente et si fâcheuse dans la terminologie employée, il serait indispensable de se mettre d'accord sur un vocabulaire technique commun et unifié31 ${ }^{31}$. Pour éviter les confusions liées à des différences de définitions, ils décident de créer un « lexique polyglotte général relatif à la conservation et à la restauration des peintures ${ }^{32}$ $»$.

Une fois les conditions du dialogue mises en place, une réflexion commune sur le nettoyage des peintures peut être développée. Cette réflexion ne pouvant être mise en place qu'en s'écartant de l'ancienne tradition des restaurateurs de garder leurs procédés secrets, elle s'appuie sur un partage des connaissances et des opinions, qui permet ainsi de confronter les pratiques pour rechercher une solution effective. Ce partage, particulièrement encensé au sein de la Commission de l'ICOM pour le traitement des peintures, s'est fait de différentes façons. Les institutions ont notamment ouvert leurs portes aux experts étrangers, en leur présentant au cours des conférences de cette commission leurs laboratoires et leurs ateliers de restauration mais aussi en explicitant leurs pratiques. Cette ouverture est redoublée par l'accueil d'étudiants ou de professionnels étrangers. La question de la formation des restaurateurs, au cœur des critiques et des débats de la « querelle des vernis », conduit en effet à promouvoir l'internationalisation de la formation: étudiants et professionnels sont encouragés à aller étudier d'autres approches et découvrir des pratiques différentes dans les institutions étrangères ou via des conférences d'experts étrangers ${ }^{33}$. Les différentes pratiques sont également partagées et diffusées au moyen d'une bonne documentation complète et précise des restaurations dont l'importance est soulignée dans les débats de la «querelle des vernis». La Commission de l'ICOM pour le traitement des peintures se penche notamment sur la question, en promulguant des recommandations comme l'inclusion dans les dossiers de documentation de photographies avant, pendant et après nettoyage et l'enregistrement photographique des examens faits sous différents rayonnements ${ }^{34}$. Elle s'applique également à regrouper les connaissances, "son premier devoir éta[n]t de réunir la plus grande masse possible de renseignements sur la pratique de la conservation et de la restauration dans les plus importants musées du monde et sur les doctrines qu'on y professait à ce sujet ${ }^{35}$ ». Pour cela, elle conçoit et diffuse des questionnaires sur les pratiques de restauration, dont un spécifiquement centré sur le dévernissage réalisé par René Huyghe ${ }^{36}$.

Les Cahiers de l'École du Louvre, 14 | 2019 
Ce groupement des connaissances permet de comparer les pratiques de nettoyage entre différents pays et vise ainsi, par cette confrontation, à définir une pratique commune susceptible de remédier aux critiques et aux polémiques résultant du nettoyage des tableaux. Toutefois, cette volonté échoue en raison de l'affirmation, par tous les acteurs du débat quelle que soit leur position, de la nécessité de prendre en compte les spécificités de chaque cas particulier et de s'y adapter.

21 Une autre solution est cependant développée dans le cadre de la " querelle des vernis ", qui a connu un grand succès et ce jusqu'à nos jours : la création de comités consultatifs internationaux pour la restauration des œuvres à risque. Cette mise en place est liée à l'apparition du principe de partage de responsabilité pour les interventions sur des biens supranationaux ${ }^{37}$. Dans le contexte de réflexions et de collaborations induites par la "querelle des vernis ", la restauration et le nettoyage du polyptyque de l'Adoration de l'Agneau mystique des frères Van Eyck sont le cadre de la mise en place du premier comité consultatif international en 1950, composé notamment d'acteurs de premier plan de la "querelle des vernis $»^{38}$, qui peut s'interpréter comme une première conséquence concrète de la controverse. Cette première collaboration autour d'un cas particulier prestigieux est une réussite, encensant ainsi le principe de coopération internationale mais aussi interdisciplinaire.

\section{Le progrès de la collaboration interdisciplinaire}

Les débats de la «querelle des vernis » ont mis en lumière des problèmes de compréhension entre spécialistes de différents domaines concernés - conservateurs, historiens de l'art, restaurateurs, scientifiques - sur lesquels reposent en partie les conflits. L'absence de dialogue et de compréhension entre les différentes disciplines concernées par le nettoyage a en effet contribué à renforcer les tensions. D'abord exclus du débat et de la commission de l'ICOM pour le traitement des vernis, les restaurateurs et les scientifiques ont finalement été intégrés aux discussions au terme d'un long processus concomitant avec la reconnaissance de l'importance des données scientifiques et techniques dans les choix de nettoyage. En conséquence s'est imposée la nécessité d'une approche interdisciplinaire.

L'affirmation et le développement du dialogue entre les spécialistes des différents domaines en restauration se sont traduits par la création de différentes institutions ${ }^{39}$. Est ainsi créé en 1950 l'Institut International de Conservation (IIC), premier établissement mettant en avant l'approche interdisciplinaire en regroupant des membres provenant de divers pays et des différents domaines de la conservationrestauration. Son action est poursuivie par la fondation du Centre international d'étude pour la conservation et la restauration des biens culturels de Rome (ICCROM) en 1959 et par la création du comité pour la conservation de l'ICOM (ICOM-CC) ${ }^{40}$ résultant de la fusion en 1965 de la commission de l'ICOM pour le traitement des peintures et du comité de l'ICOM des laboratoires de musées ${ }^{41}$. La "querelle des vernis» a ainsi contribué à la mise en place d'institutions actuelles essentielles dans le domaine de la conservation et de la restauration des biens culturels.

La "querelle des vernis » a suscité de nombreux débats, permettant de développer des connaissances sur le nettoyage des peintures et de rechercher des solutions grâce à une collaboration internationale et interdisciplinaire. Malgré les discussions, elle ne débouche pas sur une solution définitive au problème du nettoyage des tableaux et des 
controverses qui en découlent. En témoignent les récentes polémiques, comme celle autour du nettoyage de la Sainte Anne de Léonard de Vinci en 2012. Néanmoins, la "querelle des vernis" a tout de même contribué à développer et à formuler des problématiques et des notions aujourd'hui essentielles dans le domaine de la conservation-restauration, tels que le principe de prudence, l'adaptation au cas particulier, l'importance de la documentation et de la formation des conservateursrestaurateurs, la recherche d'un vocabulaire commun, la reconnaissance d'œuvres supranationales, le principe des comités consultatifs internationaux, l'application des sciences au domaine de la conservation-restauration et le dialogue international et interdisciplinaire.

\section{NOTES}

1. Voir notamment: Sheldon Keck, «Some Picture Cleaning Controversies: Past and Present", Journal of the American Institute for Conservation, vol. XXIII, $\mathrm{n}^{\circ 2}, 1984$, pp. 73-87; Ségolène Bergeon, "Polemics surrounding the restauration painting and sculpture : a short history ", Zeitschrift für Kunsttechnolohie und Konservierung, vol. XV, $\mathrm{n}^{\circ}$, janvier 2001, pp. 7-24.

2. Ces polémiques eurent respectivement pour conséquence la démission du conservateur de la National Gallery Sir Charles Eastlake (qui en devint ensuite le premier directeur en 1855) et celle du conservateur des peintures du musée du Louvre Frédéric Villot.

3. Anonyme, The National Gallery 1938-1954, Londres, Balding \& Mansell, 1955, p. 61.

4. Voir Fanny Girard, La "querelle des vernis ». Une controverse sur le nettoyage des peintures au milieu du XXe siècle, École du Louvre, mémoire de master 2, sous la direction de Delphine Burlot et Clémence Raynaud, École du Louvre, 2018: toutes les critiques publiées dans le Times sont présentées et étudiées dans les annexes.

5. David Batchelor, Chromophobia, Londres, Reaktion books, 2000.

6. John Reginald Homer Weaver, président du Trinity College d'Oxford, est une personnalité "profane" en conservation, tandis que George Leslie Stout était à l'époque chef du département de la restauration du Fogg Art Museum d'Harvard, et Paul Coremans chef du Laboratoire Central des Musées Nationaux de Belgique.

7. John Reginald Homer Weaver, George Leslie Stout, Paul Coremans, «Le rapport Weaver sur le nettoyage des peintures de la National Gallery à Londres ", Museum, vol. III, $\mathrm{n}^{\circ}$, 1950 , p. 32-72.

8. Cat. d'exp. An exhibition of cleaned pictures 1936-1947, sous la direction de Sir Philip Hendy, Londres, The National Gallery, 1947, Londres, The National Gallery, 1947.

9. Centre d'information UNESCO-ICOM, Archives de l'ICOM, Carton « Fonds ICOM ART. 108 CO65 ", Dossier «art. 108, $1^{\text {re }}$ conférence biennale Paris 1948 », Pochette "Actes imprimés »: ICOM. Première conférence biennale. Paris, 28 juin-3 juillet 1948. Résumé des travaux. Compte rendu des manifestations, p. 114. 
10. Centre d'information UNESCO-ICOM, Archives ICOM, Carton «ICOM-CC », Pochette "Commission de l'ICOM pour le traitement des peintures. Réunion de Londres 13-15 décembre 1948 National Gallery », Feuille "Thèmes de discussion ».

11. René Huyghe, «Le problème du dévernissage des peintures anciennes et le musée du Louvre ", Museum, vol. III, $\mathrm{n}^{\circ 3}$, 1950, pp. 83-90.

12. Quatre contributions paraissent tout d'abord en 1949-1950 dans le Burlington Magazine, impliquant Cesare Brandi (directeur de l'Istituto centrale del restauro de Rome), Neil MacLaren (assistant de conservation puis conservateur adjoint à la National Gallery), Anthony Werner (chercheur en chimie à la National Gallery) et Ernst Gombrich (historien de l'art, directeur du Warburg Institut). Le débat ne reprend ensuite qu'en 1961 avec un article d'Helmut Ruhemann (restaurateur en chef de la National Gallery) publié dans The British Journal of Aesthetics. Il est poursuivi en 1962 et 1963 dans une quinzaine d'articles, parus principalement dans The Burlington Magazine, de différents auteurs tels que Ernst Gombrich, Otto Kurz (historien de l'art, bibliothécaire en chef du Warburg Institute), Stephen Rees Jones (chef du département technologique du Courtauld Institut of Art), Joyce Plesters (assistante au département scientifique de la National Gallery), Denis Mahon (historien de l'art siégeant au conseil des trustees de la National Gallery) ou encore Gary Thomson (chef du département scientifique et conseiller scientifique des trustees de la National Gallery). Voir F. Girard, op. cit., note $3:$ liste chronologique des articles dans les annexes.

13. Helmut Ruhemann, The cleaning of paintings, Londres, Faber and Faber, 1968, p. 80 : «two schools of cleaning ».

14. René Huyghe semble être le premier à utiliser les termes de "totalitaires" et de "nuancés » d'après Anonyme, "The Louvre Since The War », The Times, 17 août 1950, probablement dans R. Huyghe, op. cit. note 10, p. 83. Ils sont repris par la suite dans la majorité des articles rattachés à la «querelle des vernis» et dans les textes historiographiques.

15. Voir notamment: Gilberte Emile-Mâle, Restauration des peintures de chevalet, Fribourg, Office du Livre, 1976; Gilberte Emile-Mâle, Pour une histoire de la restauration des peintures en France, Paris, Somogy, 2009 ; Cat. d'exp. Restauration des peintures, sous la direction de Ségolène Bergeon, Paris, Musée du Louvre, 30 mai- $1^{\text {er }}$ décembre 1980, Paris, Réunion des Musées Nationaux, 1980, p. 7.

16. Huyghe René, « Not if, but how ", Art and Design, vol. II, $\mathrm{n}^{\circ 1}$, mars 1947, dans J. R. H. Weaver, G. L. Stout, P. Coremans, op. cit., note 5, pp. 56,57.

17. Arthur W. Lucas, «The cleaning of pictures. Some reasons and reactions ", Apollo, vol. LXV, $\mathrm{n}^{\circ} 386$, avril 1957, p. 144.

18. Centre d'information Unesco-ICOM, Archives ICOM, Carton "ICOM-CC», Pochette "Commission de l'ICOM pour le traitement des peintures. Réunion de Londres 13-15 décembre 1948 National Gallery", Pochette "Séances de travail. Compte rendu ", pochette "Intervention des conférenciers ", Pochette " Theodore Rousseau », Rousseau Theodore, «Le nettoyage et la restauration des peintures », p. 1.

19. Id., Ibid., p. 1.

20. R. Huyghe, op. cit., note 11, p. 90.

21. Anonyme, «Le dévernissage des tableaux à la National Gallery », Museum, vol. III, $\mathrm{n}^{03}, 1950, \mathrm{p} .132$. 
22. Gerry Hedley, Measured Opinions. Collected papers on the conservation of paintings, Londres, C. Villlers United Kingdom Institute for Conservation, 1993, p. 174.

23. Archives du C2RMF, site des petites écuries de Versailles, Procès-verbaux des Commissions de restauration du 28 mars 1950, du 22 avril 1952 et du 9 juin 1949.

24. Archive Nationale, site de Pierrefitte-sur-Seine, 20150163/58, 6EE3 Correspondance 1965-1967, Lettre de Germain Bazin à Monsieur Slabczynski, [sans lieu], le 22 novembre 1967.

25. Steven W. Dykstra, «The artist's intentions and the intentional fallacy in fine arts conservation ", Journal of the American Institute for Conservation, vol. XXXV, $\mathrm{n}^{\circ 3}, 1996$, p. 202.

26. Voir notamment : Gilberte Emile-Mâle, «Conservation et restauration des peintures au musée du Louvre ", La sauvegarde de l'art français, cahier 4, 1987, p. 70 ; cat. d'exp. Restauration des peintures, sous la direction de Ségolène Bergeon, Paris, Musée du Louvre, 30 mai- $1^{\text {er }}$ décembre 1980, Paris, Réunion des Musées Nationaux, p. 7.

27. Arthur Van Schendel, « La commission de l'ICOM pour le traitement des peintures et le problème du dévernissage ", Museum, vol. IV, $\mathrm{n}^{\circ 1}$, 1951, p. 142.

28. Pierre Leveau, «La querelle des vernis et le différent des sciences et des lettres", Synergies Royaume-Uni et Irlande, $\mathrm{n}^{07}$, 2014, p. 57-69.

29. Cesare Brandi, « The cleaning of pictures in relation to patina, varnish and glazes ", The Burlington Magazine, vol. XCI, $\mathrm{n}^{0556}$, juillet 1949, p. 183-188, dans BRANDI Cesare, Théorie de la restauration, $1^{\text {re }}$ éd. Rome, Edizione di storia e letteratura, 1963, trad. de l'italien par Monique Baccelli, Paris, Allia, 2011, pp. 93-102.

30. Paul Philippot, "Le nettoyage des peintures. Réflexions critiques", 1986, dans Catheline Périer-D'Ieteren, Paul Philippot. Pénétrer l'art, restaurer l'œuvre. Une vision humaniste. Hommage en forme de florilège, Courtrai, Groeninghe, 1990, p. 407.

31. Arthur Van Schendel, « La commission de l'ICOM pour le traitement des peintures et le problème du dévernissage ", Museum, vol. IV, n ${ }^{\circ 1}, 1951$, p. 141.

32. Id., Ibid., p. 142.

33. Centre d'Information Unesco-ICOM, Archives ICOM, Carton "ICOM-CC», Philip Hendy, «Problèmes relatifs à l'entretien et à la restauration des peintures. Rapport sur les progrès réalisés dans le domaine de la coopération internationale ", Deuxième conférence biennale du Conseil International des Musées, Londres 17 au 22 juillet 1950, p. 6.

34. Id., Ibid., p. 6.

35. A. Van Schendel, op. cit., note 21, p. 141.

36. Centre d'information Unesco-ICOM, Archives de l'ICOM, Carton "ICOM-CC", Pochette « Commission de l'ICOM pour le traitement des peintures. Réunion de Londres 13 au 15 décembre 1948 National Gallery », Note de René Huyghe, signée, Paris, le 22 décembre 1948 ; Anonyme, "Le dévernissage des tableaux à la National Gallery ", Museum, vol. III, $\mathrm{n}^{\circ 3}$, 1950, p. 131.

37. Anonyme, « Editorial », Museum, vol. IV, $\mathrm{n}^{\circ 1}$, 1951, p. 137.

38. Paul Coremans, L'agneau mystique au laboratoire: examen et traitement, collection Les Primitifs Flamands, Anvers, De Sikkel, 1953, p. 8. 
39. Rebecca Rushfield, "Conservation organizations and professional standards", dans Joyce Hill Stoner, Rebecca Rushfield, Conservation of easel paintings, Londres, Routledge, 2012, p. 761.

40. Anonyme, "ICOM Committee on Conservation ", Bulletin of the American Group. International Institute for Conservation of Historic and Artistic Works, vol. VIII, $\mathrm{n}^{01}$, octobre 1967 , p. 12.

41. Archives Nationales, site de Pierrefitte-sur-Seine, 20150163/58, 6EE3, Correspondance du conservateur en chef Germain Bazin 1966, Lettre de Germain Bazin à Monsieur le Directeur des Musées de France, le 19 septembre 1967, p. 1.

\section{RÉSUMÉS}

La « querelle des vernis » s'inscrit dans une longue tradition de controverses sur le nettoyage des peintures, débats qui réapparaissent encore ponctuellement de nos jours. Fréquemment perçue comme un conflit entre deux pratiques de nettoyage, le dévernissage total et l'allègement de vernis, elle est également le cadre de profondes réflexions sur des notions telles que la prudence des interventions, la documentation, la formation des restaurateurs et l'importance du dialogue. Elle a en effet beaucoup contribué au développement de la coopération internationale et interdisciplinaire en matière de conservation et de restauration des biens culturels. Datant du milieu du XX $\mathrm{XX}^{\mathrm{e}}$ siècle et précédant directement la théorisation de la restauration moderne, cette polémique se présente comme un moment fort de l'élaboration des principes et des pratiques actuels.

The "cleaning controversy" is part of the long tradition of controversies over the cleaning of paintings, debates that still reoccur from time to time. Frequently perceived as a conflict between two cleaning practices, complete cleaning and partial cleaning, it is also the context for profound reflection on concepts such as the prudence des interventions, documentation, the training of restorers and the importance of dialogue. It greatly contributed to the development of international and interdisciplinary cooperation in the domain of the conservation and restoration of cultural goods. Dating from the mid-twentieth century and directly preceding the theorisation of modern restoration, the polemic was a moment fort in the elaboration of current principles and practices.

\section{INDEX}

Mots-clés : querelle des vernis, dévernissage, allègement, controverse internationale, Cesare Brandi, Ernst Gombrich, René Huyghe, National Gallery, ICOM, interdisciplinarité

Keywords : cleaning controversy, complete cleaning, partial cleaning, international controversy, Cesare Brandi, Ernst Gombrich, René Huyghe, National Gallery, ICOM, interdisciplinarity 


\section{AUTEUR}

\section{FANNY GIRARD}

Diplômée du deuxième cycle de l'École du Louvre (parcours recherche en histoire de l'art appliquée aux collections) en 2018, Fanny Girard s'est intéressée dans ses recherches à l'histoire de la restauration des biens culturels en étudiant la " querelle des vernis ", controverse sur le nettoyage des tableaux au milieu du XX ${ }^{\mathrm{e}}$ siècle, sous la direction de Delphine Burlot et Clémence Raynaud. Préparant actuellement les concours de recrutement des conservateurs du patrimoine, elle s'est également employée à diffuser son travail de recherche, notamment dans le cadre d'une conférence lors d'une journée d'étude (mars 2019) du groupe « Peinture de chevalet » de la SFIIC (Section Française de l'Institut International de Conservation) et d'une présentation dans le master de Conservation-restauration des biens culturels de l'Université Panthéon-Sorbonne. A graduate of the École du Louvre (research, art history applied to collections) in 2018, Fanny Girard became interested in the history of the restoration of cultural goods when studying the "cleaning controversy", a mid-twentieth-century controversy over the cleaning of paintings, supervised by Delphine Burlot and Clémence Raynaud. Now preparing for the heritage curator exams, she is also disseminating her research, notably during a lecture for a study day (March 2019) of the easel-painting group of the Section Française de l'Institut International de Conservation (SFIIC) and a presentation in the Conservation-Restoration of Cultural Goods master's programme at the Université Panthéon-Sorbonne. 